\title{
Evaluation du niveau de stress thermique par mesure de la température corporelle et du niveau d'hyperventilation chez le poulet de chair dans des conditions de production au Venezuela
}

\author{
M. Pérez ${ }^{1}$ V. De Basilio ${ }^{1 *}$ Y. Colina ${ }^{1}$ Y. Oliveros ${ }^{2}$ \\ S. Yahav ${ }^{3}$ M. Picard ${ }^{4}$ D. Bastianelli ${ }^{5}$
}

Mots-clés

Poulet de chair - Climat tropical Température corporelle - Taux de respiration - Elevage de volaille Mesure - Venezuela.

\begin{abstract}
Résumé
Les mesures répétées sur des volailles en élevage sont difficiles à mettre en œuvre à cause de la difficulté d'identification et de récupération des animaux. Une technique de suivi par isolement de petits groupes d'animaux dans des cercles situés au sein même de l'élevage est proposée. Cette méthode a été utilisée dans un poulailler de production au Venezuela pour déterminer la variation de la température corporelle (TC) et du niveau d'hyperventilation $(\mathrm{NH})$, en fonction de la localisation dans le bâtiment et du sexe des animaux. Trois cercles grillagés ont été aménagés et divisés en deux, avec dans chaque demi-cercle 20 poulets mâles ou femelles. A partir du 29 e jour d'âge, la température ambiante (TA) et l'humidité relative (HR) ont été enregistrées en continu, et la TC (côlon terminal) et le NH ont été mesurés chaque heure, de 9 h 00 à 19 h 00, un jour sur deux pendant deux semaines. Les mesures de la TC et du NH ont varié au cours de la journée, sans être rigoureusement parallèles aux paramètres climatiques TA et HR. Il y a eu des différences significatives entre les cercles pour tous les paramètres mesurés. L'effet du sexe n'a pas été significatif sur la TC $(p=0,06)$, mais il l'a été sur le $\mathrm{NH}(p=0,01)$ et I'interaction avec la localisation du cercle $(p<0,001$ pour la TC et $p<0,01$ pour le $\mathrm{NH}$ ). Le sexe a eu un effet significatif sur la consommation alimentaire ( $p<0,01)$ et le gain de poids $(p=0,02)$ mais pas sur l'indice de consommation $(p=0,64)$. Les mesures climatiques ont également été différentes entre cercles et entre sexes, avec notamment une HR plus élevée près des mâles $(p<0,001)$. Sur l'ensemble des données, la TC et le NH ont augmenté respectivement de $0,139{ }^{\circ} \mathrm{C}$ et 12,1 inspirations/min par degré d'augmentation de la TA. Il est conclu que les conditions climatiques présentent une hétérogénéité au sein du bâtiment, avec des conséquences sur le confort des poulets, et que les mesures sur animaux (TC et $\mathrm{NH}$ ) doivent compléter les données climatiques pour décrire l'état de stress thermique. La technique expérimentale proposée permet d'observer des animaux de manière répétée dans les conditions réelles d'un poulailler de production.
\end{abstract}

1. Instituto de Producción Animal, Maracay 2101, Venezuela.

2. Instituto Nacional de Investigaciones Agricolas, Maracay 2101, Venezuela.

3. Institute of Animal Science, Bet-Dagan, 50250, Israel.

4. Inra, Station de recherches avicoles, Nouzilly, F-37380 France.

5. Cirad, UPR Systèmes d'élevage, Montpellier, F-34398 France.

* Auteur pour la correspondance

Instituto de Producción Animal, Facultad de Agronomía, UCV, Apdo. Postal 4579,

Maracay 2101, Venezuela.

E-mail : vascodebasilio@hotmail.com

\section{INTRODUCTION}

L'aviculture a connu un essor extrêmement important dans les pays tropicaux ces dernières années. Au Venezuela, la consommation de viande de volaille a atteint $26 \mathrm{~kg} / \mathrm{an} /$ personne en $2005(14,15)$, représentant presque 60 p. 100 des protéines carnées consommées. Outre les problèmes économiques vénézuéliens, la production doit faire face à un environnement thermique contraignant puisque la moitié des exploitations avicoles se situent dans des régions où la température moyenne annuelle est supérieure à $30^{\circ} \mathrm{C}$, avec des maxima supérieurs à $36{ }^{\circ} \mathrm{C}$ pendant plusieurs heures de la journée (1). De telles 
températures peuvent provoquer des coups de chaleur susceptibles de causer des mortalités pouvant concerner la moitié des animaux (23), même en utilisant des densités d'élevages modérées (environ 10 ani$\operatorname{maux} / \mathrm{m}^{2}$ ), notamment dans le contexte vénézuélien où l'humidité relative est élevée (généralement 75 à 100 p. 100). Les pertes économiques sont très importantes compte tenu du fait que les mortalités par coup de chaleur surviennent en fin de période d'élevage, alors que les animaux ont déjà consommé plus de 80 p. 100 de l'aliment et sont quasiment prêts à la commercialisation (1). Les coups de chaleur sont difficiles à prévoir d'après les informations météorologiques, mais ils surviennent quand les températures sont élevées $\left(32^{\circ} \mathrm{C}\right)$ pendant de longues périodes ( $>6$ heures) ou très élevées $\left(36^{\circ} \mathrm{C}\right)$ pendant des périodes plus courtes ( $>2$ heures) (7).

L'utilisation de pratiques d'élevage adaptées pour diminuer les effets négatifs du stress thermique dans les élevages de poulets de chair serait plus efficace si l'on pouvait prédire le niveau de stress thermique et le risque de mortalité avec des indicateurs faciles à mesurer. Par exemple, on sait que l'utilisation de la restriction alimentaire durant les heures les plus chaudes de la journée peut limiter le stress thermique $(4,18)$. Cette pratique tend à réduire le gain de poids quand elle est utilisée dans les deux dernières semaines d'élevage, sauf si elle est mise en œuvre uniquement pendant la dernière semaine (21). Un suivi du niveau de stress thermique pendant les deux dernières semaines permettrait de n'appliquer la restriction alimentaire que s'il existe un risque immédiat de mort par coup de chaleur, et donc d'optimiser à la fois la survie et les performances.

Bien qu'on ne connaisse pas exactement le détail des processus physiologiques qui conduisent à la mort des poulets (9), il existe deux paramètres facilement mesurables qui changent chez les animaux en stress thermique : la fréquence respiratoire et la température corporelle (13). La fréquence respiratoire, appelée ici niveau d'hyperventilation $(\mathrm{NH})$ dès lors que la respiration accélérée s'accompagne de l'ouverture du bec, augmente de 25 inspirations par minute (insp/min) à la thermoneutralité [température ambiante $\left.(\mathrm{TA})<22{ }^{\circ} \mathrm{C}\right]$ à $200(13)$, voire $300 \mathrm{insp} / \mathrm{min}$ (26) quand la température ambiante dépasse $36{ }^{\circ} \mathrm{C}$. La température corporelle (TC) augmente de $41,5^{\circ} \mathrm{C}$ (TC normale) à $47^{\circ} \mathrm{C}$ (TC au moment de la mort par chaleur) (6).

Ces indicateurs ont été définis dans des conditions de laboratoire, avec un nombre restreint d'animaux (26), avec une température ambiante contrôlée strictement et des conditions d'élevage (densités d'animaux, conditions sanitaires) très différentes des pratiques réelles d'élevage. Des validations effectuées dans des conditions réelles sont donc nécessaires pour pouvoir les proposer comme outils utilisables sur le terrain pour des expérimentations ou, à terme, pour la caractérisation de l'état de stress des animaux en vue de la gestion technique des élevages.

La réalisation de telles validations sur le terrain se heurte à des difficultés expérimentales. En effet, l'interprétation de données comme la $\mathrm{TC}$ et le $\mathrm{NH}$, qui présentent une forte variabilité individuelle, nécessite un suivi des animaux dans le temps. Or dans un poulailler commercial comportant des milliers d'animaux, l'identification des animaux est extrêmement difficile. Des marquages avec des couleurs ou avec la section de l'extrémité d'un doigt (8) ont été employés pour suivre des animaux relâchés au sein du poulailler, mais les animaux sont néanmoins difficiles à repérer. Leur capture est longue [plusieurs heures avec marquage coloré (8)], ce qui ne permet pas une stabilité des conditions environnementales pendant une session de mesures, et une nouvelle capture de tous les animaux n'est pas obligatoirement assuree [240 animaux sur 960 avec marquage par section d'un doigt (8)]. D'autre part, le stress des animaux dû au dérangement pendant leur recherche et à la capture elle-même est important, perturbant les mesures de la $\mathrm{TC}$ et du NH. Ces solutions de marquages ne sont donc pas très satisfaisantes dans la pratique.

Un des objectifs du présent travail a été la mise au point d'une technique permettant d'effectuer des mesures individuelles répétées sur des animaux placés dans des conditions réelles d'élevage. Pour ce faire, les auteurs ont choisi d'adapter la technique de cloisonnement d'animaux au sein de l'élevage, développée pour le conditionnement thermique des poussins $(7,8)$.

Cette technique a été appliquée ici à l'évaluation de la TC et du $\mathrm{NH}$ comme indicateurs du niveau de stress des poulets de chair en fin de cycle, en fonction du sexe et en relation avec les variations naturelles de la TA et de l'humidité relative (HR) pendant la journée, dans des conditions commerciales : poulaillers ouverts, densité modérée ( 7 animaux $\left./ \mathrm{m}^{2}\right)$ et aliment commercial.

\section{MATERIEL ET METHODES}

\section{Localisation de l'essai}

L'expérience s'est déroulée dans l'élevage avicole Santa María, entre les communes de Vila de Cura (district de Zamora) et Cagua (district de Sucre) dans l'Etat d'Aragua, au Venezuela (figure 1). L'HR moyenne est de 76 p. 100, respectivement avec des minima et des maxima de 52 et $99 \mathrm{p}$. 100, et la température moyenne est de $26,6{ }^{\circ} \mathrm{C}$ respectivement avec des minima et des maxima de 16,0 et $36,3{ }^{\circ} \mathrm{C}$. La valeur d'évaporation potentielle est de $1369 \mathrm{~mm}$ avec des précipitations annuelles de $834 \mathrm{~mm}$.

La période sèche s'étale de novembre à avril et les mois humides de mai à octobre. La chaleur est maximale à la fin de la période sèche (mars-avril, moyennes de $34-36^{\circ} \mathrm{C}$ ) et les valeurs minimales au début de la période sèche (novembre-décembre, moyennes de 26-28 ${ }^{\circ} \mathrm{C}$ ). L'expérimentation a eu lieu au mois de juillet, période durant laquelle la température moyenne est de $32{ }^{\circ} \mathrm{C}$ environ.

\section{Bâtiments}

L'élevage possédait 14 poulaillers, répartis par groupes de deux, séparés de $30 \mathrm{~m}$. Les bâtiments étaient tous similaires. Leur surface était de $1240 \mathrm{~m}^{2}$ (124 m sur $10 \mathrm{~m}$ ), la hauteur de 4,0 $\mathrm{m}$ au centre et de 3,0 m sur les côtés. Les toits étaient couverts de tôle avec une pente de 20 p. 100 et un débordement de 1,10 m. Les parois

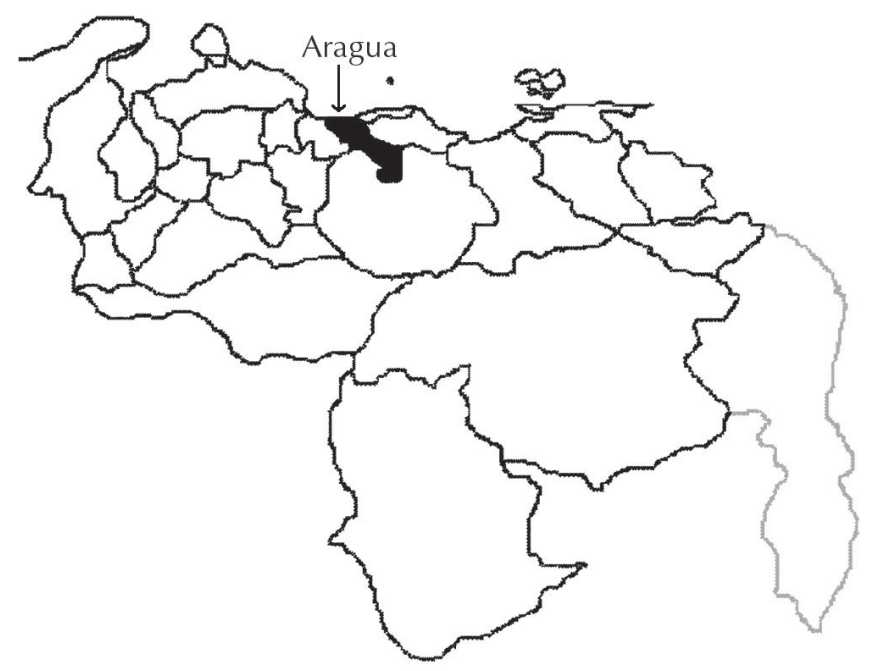

Figure 1 : localisation de l'Etat d'Aragua au Venezuela. 
du poulailler consistaient en $45 \mathrm{~cm}$ de muret surmonté de grillage. Le sol était en ciment, avec une litière de balles de riz de $8 \mathrm{~cm}$ d'épaisseur.

L'aliment était conservé dans des silos de type tour de $12,5 \mathrm{t}$ et l'eau dans des réservoirs de 2000 l. L'essai s'est déroulé dans le poulailler $\mathrm{n}^{\circ} 2$. Il comprenait trois rangées de mangeoires manuelles suspendues, espacées de 1,30 m (93 mangeoires par rangée, soit 279 au total) et deux rangées d'abreuvoirs automatiques, type Plasson, espacés de 2 m (62 abreuvoirs par rangée, soit 124 au total).

\section{Installations expérimentales}

Les animaux expérimentaux ont été placés dans des cercles grillagés (mailles de 1,5 cm sur 8,0 cm). Un cercle était formé de trois plaques de grillage dont deux étaient attachées pour former le cercle proprement dit et la troisième servait de cloison délimitant deux demi-cercles. Trois cercles ont été mis en place une semaine avant l'essai ( $21^{\mathrm{e}}$ jour des poulets). Dans chaque demi-cercle ont été placés un abreuvoir et une mangeoire suspendue, identiques à ceux utilisés dans le reste du bâtiment. Les cercles ont été placés au centre du poulailler et de chaque côté (figure 2), de manière à pouvoir étudier l'effet de leur localisation sur les résultats. Le premier cercle était à 28,0 m par rapport à l'avant du bâtiment et

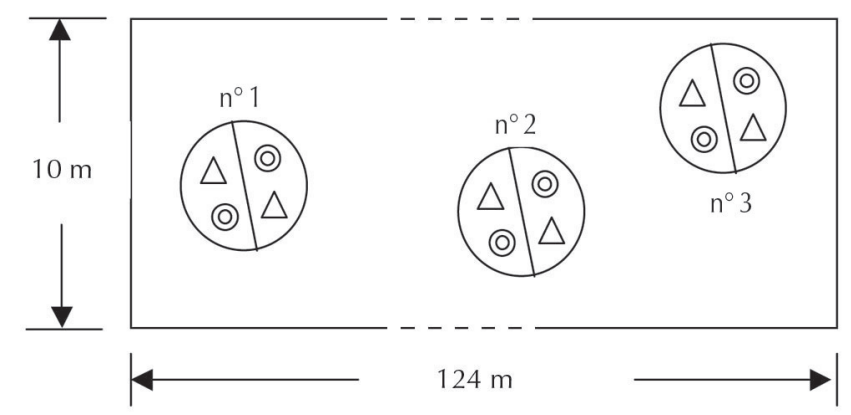

$\triangle$ Abreuvoir $\quad$ () Mangeoire

Figure 2 : répartition des cercles dans le poulailler. à $5,5 \mathrm{~m}$ par rapport au côté, le second respectivement à 69,0 et à $6,4 \mathrm{~m}$, et le troisième respectivement à 118,0 et à $2,6 \mathrm{~m}$. La surface des cercles a été ajustée de manière à ce que les animaux aient la même densité ( 7 animaux $/ \mathrm{m}^{2}$ ) que dans le reste du bâtiment. Ce système a permis d'avoir des animaux expérimentaux placés dans les mêmes conditions que celles de l'élevage, mis à part la liberté de se déplacer au-delà du cercle. Une illustration du dispositif est présentée à la figure 3 .

\section{Equipements}

Pour mesurer la TA $\left({ }^{\circ} \mathrm{C}\right)$ et l'HR (\%) du poulailler et de l'extérieur, deux thermohydrographes à tambour de marque Siap ont été utilisés. Pour enregistrer les variations ponctuelles au niveau des cercles pendant les mesures de $\mathrm{TC}$ et de $\mathrm{NH}$, un thermohygromètre digital portable de marque Tannus à lecture rapide et de précision de lecture à $0,1^{\circ} \mathrm{C}$ et 1 p. $100 \mathrm{HR}$ a été utilisé. Cet instrument a été positionné dans le cercle étudié au début de chaque série de mesures. La sonde a été placée près du sol, de manière à capter les conditions climatiques existant au niveau des animaux. La TC a été mesurée avec un thermomètre Testo 110 , avec une sonde d'immersion-pénétration d'une précision de mesure de $0,1{ }^{\circ} \mathrm{C}$. Un chronomètre de marque Casio $(0,01 \mathrm{~s})$ a été utilisé pour la mesure des durées d'hyperventilation. Une balance Mettler (de 0 à $5000 \mathrm{~g}$, précision de mesure $0,1 \mathrm{~g}$ ) a été utilisée pour peser les aliments et les animaux.

\section{Eclairage}

Pendant tout l'essai, les animaux ont été éclairés 24 heures sur 24. Pendant la nuit, un éclairage permanent a été assuré par des ampoules de lumière blanche $(100 \mathrm{~W})$ disposées à une hauteur de $3 \mathrm{~m}$, au centre du bâtiment : tous les quatre mètres dans la zone de réception des animaux, et tous les huit mètres dans le reste du bâtiment (éclairement au niveau des animaux $=30$ lux ).

\section{Animaux}

Cent vingt poulets de chair hybrides Ross x Ross ont été utilisés. Ils ont été choisis aléatoirement parmi les 9600 animaux présents dans le bâtiment, de manière à obtenir 60 (3 groupes de 20) mâles et 60 (3 groupes de 20) femelles. Chaque cercle expérimental a
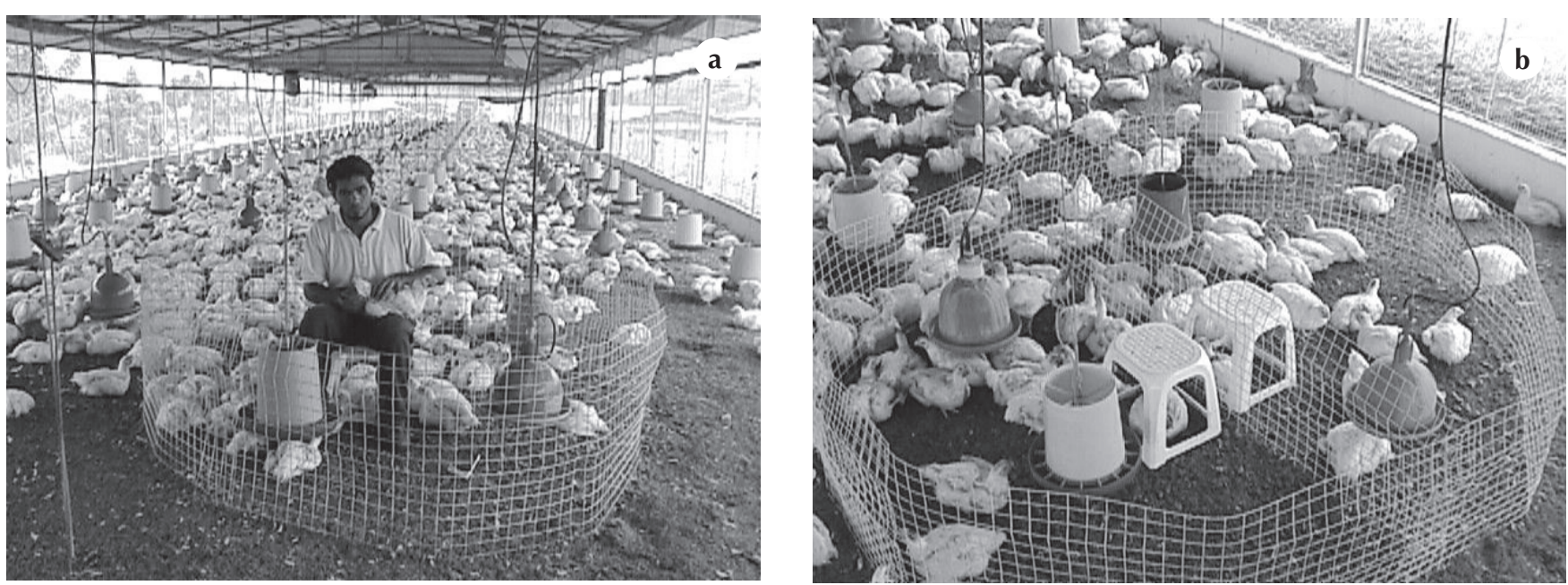

Figure 3 : dispositif expérimental ; a) Cercle d'isolement au sein de l'élevage et opérateur ; b) Détail d'un cercle d'isolement avec deux demi-cercles. 
donc été séparé en deux et a reçu un groupe de 20 mâles d'un côté et un groupe de 20 femelles de l'autre. Les animaux ont été identifiés par un marquage de couleur, avec un codage par la combinaison de cinq couleurs en trois endroits du corps : tête, dos et ailes.

\section{Prophylaxie}

Les poussins ont été vaccinés au couvoir (vaccins huileux) contre la maladie de Gumboro (Bursal suave), la bronchite infectieuse et la maladie de Newcastle (La Sota). Entre trois et cinq jours d'âge, les animaux ont reçu un traitement à la phosphomycine-fructose + biphosphate + vitamine E et une supplémentation en électrolytes dans l'eau de boisson ( $8 \mathrm{mg}$ par poussin le $3^{\mathrm{e}}$ jour et $5 \mathrm{mg}$ par poussin les $4^{\mathrm{e}}$ et $5^{\mathrm{e}}$ jours). Le $7^{\mathrm{e}}$ jour une vaccination contre la maladie de Newcastle (La Sota) et la maladie de Gumboro (Bursine) a été réalisée en nébulisation (grosses gouttes). Le 17 e jour, des rappels contre la maladie de Newcastle (Avinew) et la maladie de Gumboro (Bursine II) ont été faits en nébulisation (gouttes fines). Le $19^{\mathrm{e}}$ jour tous les animaux ont été traités avec du tartrate de tylosine (35 mg/poulet dans l'eau de boisson).

\section{Aliments}

Les aliments distribués dans les cercles ont été les mêmes aliments commerciaux que ceux de l'élevage, marque La Caridad (Alimentos La Caridad, C.A. Valencia, Edo, Carabobo, Venezuela). Avant la période expérimentale, les animaux ont été nourris avec un aliment de démarrage en miettes. Pendant l'expérimentation, ont été utilisés un aliment de croissance de 28 à 35 jours (granulé concassé, $\mathrm{MAT}^{*}=20-23$ p. $100, \mathrm{EM}^{* *}=3300 \mathrm{kcal} / \mathrm{kg}$ ) et un aliment finition de 36 à 42 jours (granulé, MAT = 19-21 p. 100, EM = 3425 $\mathrm{kcal} / \mathrm{kg})$. L'eau de boisson a été traitée au chlore $(0,1 \mathrm{~g} / \mathrm{l})$.

\section{Traitements}

L'étude a porté sur des comparaisons entre mâles et femelles et l'effet de la localisation dans le bâtiment (trois cercles). Chaque modalité a comporté 20 répétitions (animaux) pour les mesures individuelles (TC, $\mathrm{NH}$ et poids vif). Pour les mesures de consommation alimentaire et indice de consommation, l'effet du sexe n'a été étudié qu'avec trois répétitions (demi-cercles).

\section{Variables mesurées}

\section{Température corporelle}

La TC a été mesurée tous les deux jours entre 29 et 39 jours d'âge et à 42 jours. La mesure individuelle de la TC a été réalisée par introduction de la sonde dans le cloaque du poulet jusqu' au côlon terminal, comme préconisé par De Basilio et coll. (8) $(4 \mathrm{~cm}$ de pénétration de 29 à 35 jours d'âge et $5 \mathrm{~cm}$ de 37 à 42 jours). La lecture a été réalisée après stabilisation de l'instrument (approximativement $10 \mathrm{~s}$ ). Pour la mesure, l'opérateur était situé au centre du cercle en permanence, ce qui lui permettait d'attraper les oiseaux en tendant simplement les bras (figure 3), évitant ainsi toute manipulation brutale qui aurait causé du stress et donc interféré avec les résultats. Le temps total de mesure a été d'environ une minute par animal. Quatre séries de mesure par jour ont été réalisées pour tous les animaux (de 8 h 00 à 18 h 30, séparées de $30 \mathrm{~min}$ ), en changeant chaque fois l'ordre dans lequel les cercles étaient abordés.

\section{Niveau d'hyperventilation}

L'hyperventilation est définie par un rythme respiratoire augmenté par rapport à la thermoneutralité, avec ouverture du bec en continu

* Matières azotées totales. ${ }^{* *}$ Energie métabolisable pendant la respiration (7). Le NH est défini comme étant le rythme respiratoire dans les phases d'hyperventilation. Il a été mesuré tous les deux jours entre 32 et 40 jours d'âge et à 41 jours. Il a été déterminé en mesurant le temps $\left(\mathrm{t}_{10}\right)$ nécessaire à 10 inspirations successives au cours d'une séquence d'hyperventilation, déterminée par l'ouverture du bec. Le nombre d'inspirations par minute peut ensuite être calculé simplement $\left(\mathrm{NH}=10 * 60 / \mathrm{t}_{10}\right)$. Le temps nécessaire à la mesure du $\mathrm{NH}$ est environ de $30 \mathrm{~s}$ par animal. Six séries de mesure par jour ont été réalisées sur tous les animaux (de 8 h 00 à 19 h 40, séparées de $20 \mathrm{~min}$ ), en changeant chaque fois l'ordre dans lequel les cercles étaient abordés.

\section{Température ambiante et humidité relative}

Au début de chaque série de mesures dans un demi-cercle, la TA et l'HR ont été enregistrées à l'aide du thermohygromètre portable placé au niveau des animaux du demi-cercle. Pour pouvoir comparer les conditions climatiques à l'intérieur et à l'extérieur du bâtiment, deux thermohydrographes ont été localisés l'un au centre du poulailler, au niveau des animaux, et l'autre dehors, à l'air libre à l'ombre.

\section{Consommation d'aliments, poids vif et mortalité}

La consommation d'aliments (mesure quotidienne) et le poids vif (PV) des animaux (mesure hebdomadaire) ont été déterminés par pesée individuelle avec une balance électronique. La mortalité a été enregistrée quotidiennement tout au long de l'essai.

\section{Analyses statistiques}

Toutes les variables mesurées ont été soumises à une analyse de variance Anova en mesures répétées avec le logiciel Statview (SAS Institute, Cary, NC, USA), en prenant en compte le sexe (tableau I), ou le sexe et le cercle de mesure (tableau II). Dans les cas où des différences significatives étaient mises en évidence $(\mathrm{p}<0,05)$, des tests de Dunnet étaient réalisés pour tester la séparation des différentes modalités. L'unité expérimentale a été l'animal pour les mesures de la TC, du NH, du PV, et le demi-cercle pour les mesures de consommation et d'indice de consommation. Les relations entre la TC et la TA, d'une part, et le NH et la TA, d'autre part, ont été testées par des analyses de covariance incluant l'animal comme facteur (120 modalités).

\section{Tableau I}

Valeurs moyennes des mesures de consommation, de gain de poids et indices de consommation pendant les deux semaines d'expérimentation en fonction du sexe

\begin{tabular}{lcccr} 
& $\begin{array}{c}\text { Période } \\
\text { (jours) }\end{array}$ & $\begin{array}{c}\text { Femelles } \\
(\mathbf{g})\end{array}$ & $\begin{array}{c}\text { Mâles } \\
(\mathbf{g})\end{array}$ & $\mathbf{P}$ \\
\hline $\begin{array}{l}\text { Consommation } \\
\text { d'aliment }\end{array}$ & $29-35$ & $\begin{array}{l}891 \pm 45 \\
\text { 36-42 }\end{array}$ & $\begin{array}{l}866 \pm 9 \\
1017 \pm 25\end{array}$ & 0,07 \\
& $29-42$ & $1757 \pm 36$ & $2148 \pm 57$ & $<0,01$ \\
\hline Gain de poids & $29-35$ & $463 \pm 8$ & $572 \pm 12$ & $<0,01$ \\
& $36-42$ & $329 \pm 42$ & $426 \pm 16$ & 0,10 \\
& $29-42$ & $794 \pm 48$ & $1012 \pm 27$ & 0,02 \\
\hline Indice de & $29-35$ & $1,93 \pm 0,13$ & $1,78 \pm 0,07$ & 0,36 \\
consommation & $36-42$ & $2,73 \pm 0,37$ & $2,67 \pm 0,17$ & 0,89 \\
& $29-42$ & $2,24 \pm 0,18$ & $2,13 \pm 0,11$ & 0,64
\end{tabular}




\section{Tableau II}

Température corporelle (TC), niveau d'hyperventilation (NH), gain de poids (GP) des animaux, températures ambiantes (TA) et humidités relatives (HR) dans les cercles pendant les deux semaines d'expérimentation en fonction de la localisation dans le poulailler (cercles 1, 2, 3) et du sexe $(F, M)$

\begin{tabular}{|c|c|c|c|c|c|c|c|c|c|c|}
\hline & \multirow{2}{*}{$\begin{array}{l}\text { Période } \\
\text { (jours) }\end{array}$} & \multicolumn{2}{|c|}{ Cercle 1} & \multicolumn{2}{|c|}{ Cercle 2} & \multicolumn{2}{|c|}{ Cercle 3} & \multirow{2}{*}{$\begin{array}{c}\text { Effet cercle } \\
\text { P }\end{array}$} & \multirow{2}{*}{$\begin{array}{c}\text { Effet sexe } \\
\text { P }\end{array}$} & \multirow{2}{*}{$\begin{array}{c}\text { Interaction } \\
\mathbf{P}\end{array}$} \\
\hline & & $F$ & $M$ & $F$ & $M$ & $\mathbf{F}$ & $M$ & & & \\
\hline \multirow[t]{3}{*}{ TC } & $29-35$ & $41,71^{b}$ & $41,71^{b}$ & $41,87^{a}$ & $41,84^{\mathrm{a}}$ & $41,61^{\mathrm{c}}$ & $41,75^{b}$ & $<0,01$ & 0,07 & $<0,01$ \\
\hline & $36-42$ & $41,82^{\mathrm{C}}$ & $41,82^{\mathrm{C}}$ & $42,07^{a}$ & $41,98^{a b}$ & $41,79^{c}$ & $41,96^{b}$ & $<0,01$ & 0,31 & $<0,01$ \\
\hline & $29-42$ & $41,76^{c}$ & $41,76^{\mathrm{c}}$ & $41,96^{\mathrm{a}}$ & $41,90^{\mathrm{ab}}$ & $41,70^{d}$ & $41,85^{b}$ & $<0,01$ & 0,06 & $<0,01$ \\
\hline $\begin{array}{l}\text { TA pendant } \\
\text { mesures de TC }\end{array}$ & & $30,0^{\mathrm{C}}$ & $30,4^{b}$ & $30,7^{a}$ & $30,7^{a}$ & $30,0^{c}$ & $30,3^{b}$ & $<0,01$ & $<0,01$ & 0,05 \\
\hline $\begin{array}{l}\text { HR pendant } \\
\text { mesures TC }\end{array}$ & & $81,2^{\mathrm{C}}$ & $87,1^{\mathrm{a}}$ & $80,3^{\mathrm{c}}$ & $84,3^{b}$ & $78,7^{d}$ & $87,7^{\mathrm{a}}$ & $<0,01$ & $<0,01$ & 0,05 \\
\hline \multirow[t]{3}{*}{$\mathrm{NH}$} & $29-35$ & $134^{\mathrm{a}}$ & $118^{b}$ & $141^{\mathrm{a}}$ & $141^{\mathrm{a}}$ & $140^{\mathrm{a}}$ & $141^{\mathrm{a}}$ & $<0,01$ & 0,16 & 0,08 \\
\hline & $36-42$ & $118^{b}$ & $108^{\mathrm{c}}$ & $106^{\mathrm{c}}$ & $109^{c}$ & $127^{\mathrm{a}}$ & $120^{\mathrm{b}}$ & $<0,01$ & 0,02 & 0,01 \\
\hline & $29-42$ & $121^{b}$ & $110^{c}$ & $111^{\mathrm{c}}$ & $114^{\mathrm{C}}$ & $130^{\mathrm{a}}$ & $124^{\mathrm{ab}}$ & $<0,01$ & 0,01 & $<0,01$ \\
\hline $\begin{array}{l}\text { TA pendant } \\
\text { mesures de } \mathrm{NH}\end{array}$ & & $29,8^{c}$ & $29,7^{c}$ & $30,3^{a b}$ & $30,5^{\mathrm{a}}$ & $29,6^{c}$ & $30,1^{b}$ & $<0,01$ & 0,01 & 0,02 \\
\hline $\begin{array}{l}\text { HR pendant } \\
\text { mesures } \mathrm{NH}\end{array}$ & & $75,8^{b}$ & $76,9^{b}$ & $76,4^{b}$ & $81,0^{\mathrm{a}}$ & $76,9^{b}$ & $80,8^{a}$ & $<0,01$ & $<0,01$ & $<0,01$ \\
\hline \multirow[t]{3}{*}{ GP } & $29-35$ & $472^{b}$ & $595^{\mathrm{a}}$ & $447^{b}$ & $560^{\mathrm{a}}$ & $469^{b}$ & $560^{a}$ & 0,47 & $<0,01$ & 0,80 \\
\hline & $36-42$ & $386^{a b}$ & $448^{\mathrm{a}}$ & $247^{c}$ & $434^{\mathrm{ab}}$ & $354^{b}$ & $396^{a b}$ & 0,04 & $<0,01$ & 0,04 \\
\hline & $29-42$ & $859^{b c}$ & $1043^{a}$ & $699^{d}$ & $1037^{a}$ & $823^{c}$ & $958^{a b}$ & 0,08 & $<0,01$ & 0,04 \\
\hline
\end{tabular}

$\mathrm{a}, \mathrm{b}, \mathrm{c}, \mathrm{d}$ Les lettres différentes sur une même ligne indiquent des valeurs significativement différentes $(\mathrm{p}<0,05)$

\section{RESULTATS}

\section{Variations des conditions environnementales et effet sur les paramètres mesurés}

Les TA et HR moyennes mesurées à l'extérieur des poulaillers pendant toute la période expérimentale ont été respectivement de $24,7{ }^{\circ} \mathrm{C}$ et 81,0 p. 100 , c'est-à-dire $-2,0^{\circ} \mathrm{C}$ et $-2,6$ p. 100 par rapport à celles mesurées à l'intérieur du poulailler $\left(26,7^{\circ} \mathrm{C}\right.$ et 78,4 p. 100), la mesure ayant été réalisée avec des thermohydrographes identiques. Les températures maximales et minimales relevées ont également été plus faibles à l'extérieur $\left(32,8{ }^{\circ} \mathrm{C}\right.$ et $\left.19,6^{\circ} \mathrm{C}\right)$ qu'à l'intérieur $\left(36,0^{\circ} \mathrm{C}\right.$ et $\left.21,0^{\circ} \mathrm{C}\right)$ du bâtiment.

La TA relevée dans les cercles pendant les mesures de la TC et du $\mathrm{NH}$ (de 8 h 00 à 18 h 00 ) a été en moyenne de $30,2{ }^{\circ} \mathrm{C}$, logiquement plus élevée que la mesure globale faite sur l'ensemble du nycthémère. Elle a été significativement plus élevée dans le cercle 2 , situé au centre du bâtiment $\left(30,5 \pm 0,04{ }^{\circ} \mathrm{C}\right)$ que dans les deux autres cercles $\left(\Delta \mathrm{TA}: 0,50{ }^{\circ} \mathrm{C}\right)$, eux-mêmes significativement non différents entre eux. L'HR moyenne relevée dans la même période a été de 80,6 p. 100, sans que les différences entre cercles n'aient été significatives. Les valeurs moyennes de la TC les plus élevées ont été observées dans le cercle $2\left(41,93 \pm 0,02{ }^{\circ} \mathrm{C}\right)$, significativement plus élevées $\left(\right.$ de $0,17^{\circ} \mathrm{C}$ ) que celles des cercles 1 et 3 . Le NH observé dans le cercle 3 a été significativement plus élevé respectivement de 12 et $14 \mathrm{insp} / \mathrm{min}$ que dans les cercles 1 et 2 .

Les évolutions de la $\mathrm{TC}$ et du NH au cours de la journée sont montrées en parallèle avec les variations de la TA et de l'HR sur les figures 4 et 5 . Il s'agit des moyennes par heure des valeurs obtenues pour les différents jours de mesure, tous cercles confondus. Comme la TC et le NH n'ont pas été mesurés les mêmes jours, les valeurs de la TA et de l'HR correspondantes ont été un peu différentes. Les tendances observées ont cependant été les mêmes, avec pour la TA une phase croissante en début de journée conduisant à une élévation de température de $2{ }^{\circ} \mathrm{C}$ environ, suivie d'une stagnation ou d'une baisse de

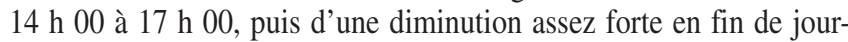
née avec une température à $19 \mathrm{~h} 00$ environ $4{ }^{\circ} \mathrm{C}$ au-dessous de la température à $12 \mathrm{~h} 00-13 \mathrm{~h} 00$. La température a été plus basse à 19 h 00 qu'à 9 h 00, ce qui signifiait que la température lors du début des mesures à $9 \mathrm{~h}$ était déjà plus élevée que la température nocturne.

L'HR a suivi une tendance inverse à la TA, mais avec deux profils différents selon les jours de mesure : sur la moyenne des jours de mesure de la TC (figure 4), la courbe a été décroissante de $8 \mathrm{~h} 00$ à 14 h 00 (de 90 à 77,5 p. 100 HR), puis croissante de 14 h 00 à $19 \mathrm{~h} \mathrm{00.} \mathrm{Sur} \mathrm{la} \mathrm{moyenne} \mathrm{des} \mathrm{jours} \mathrm{de} \mathrm{mesure} \mathrm{du} \mathrm{NH} \mathrm{(figure}$ 5), l'HR a diminué brusquement de 9 h 00 à 11 h 00 (de 87 à 75 p. 100), puis a été stable de 11 h 00 à 17 h 00, a enfin augmenté brusquement de 17 h 00 à 19 h 00 (de 72,5 à 95,0 p. 100).

La figure 4 montre les variations de la TC en parallèle avec celles de la TA et de l'HR. Une phase d'augmentation de la TC entre $9 \mathrm{~h} 00$ et $13 \mathrm{~h} 00\left(41,5^{\circ} \mathrm{C}\right.$ à $\left.42,2^{\circ} \mathrm{C}\right)$ a été suivie d'une diminution régulière de $13 \mathrm{~h} 00$ à $18 \mathrm{~h} 00\left(41,8^{\circ} \mathrm{C}\right)$ et d'une chute à $19 \mathrm{~h} 00$ $\left(41,4^{\circ} \mathrm{C}\right)$. A la fin des mesures $(19 \mathrm{~h} 00)$, la TC a été sensiblement la même qu'à 9 h $00\left(41,4{ }^{\circ} \mathrm{C}\right.$ vs $\left.41,5^{\circ} \mathrm{C}\right)$, alors que la TA a été bien inférieure. Il était également remarquable que la $\mathrm{TC}$ ait déjà diminué entre 13 h 00 et 14 h 00 alors que la TA était encore à son maximum. Toutefois ces valeurs étaient des moyennes faites sur plusieurs jours. 

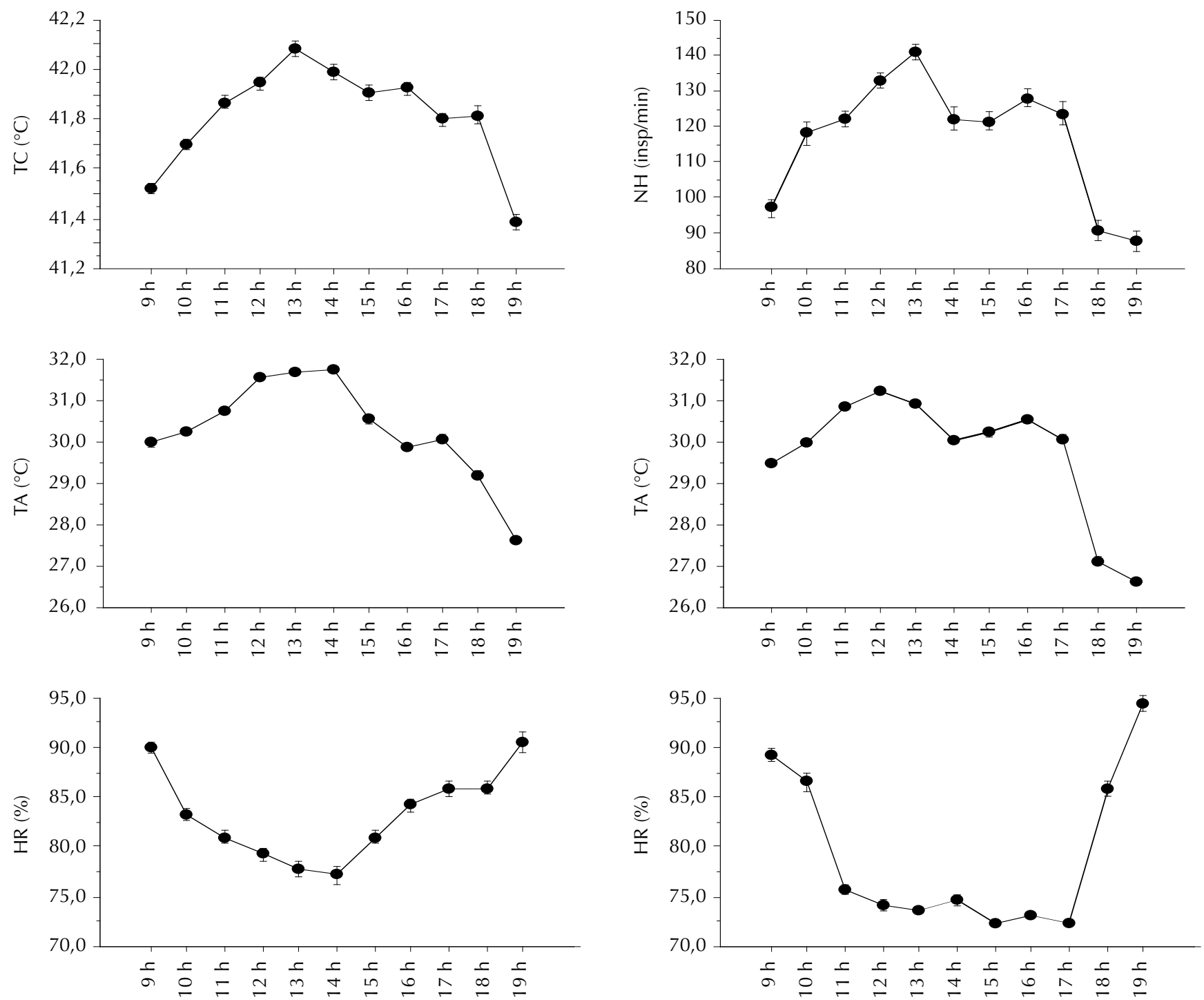

Figure 4 : variation de la température corporelle (TC), de la température ambiante (TA) et de l'humidité relative (HR) au cours de la journée. Moyenne des données de tous les jours de mesures de TC (jours 29, 31, 33, 35, 37, 39, 42).

La figure 5 montre les variations du $\mathrm{NH}$ en parallèle à celles de la TA et de l'HR. La courbe du NH a présenté une augmentation forte entre 9 h 00 et 13 h 00 (de 95 à 140 insp/min). De 12 h 00 à $13 \mathrm{~h} 00$, le NH a augmenté encore alors que la TA a stagné (ou a baissé légèrement). Le $\mathrm{NH}$ a chuté entre $13 \mathrm{~h} 00$ et $14 \mathrm{~h} 00$, puis s'est stabilisé autour de 125 insp/min de 14 h 00 à 17 h 00, avant une diminution brusque conduisant à des valeurs proches de 90 insp/min à 18 h 00 - 19 h 00, équivalentes à celles de 9 h 00 .

Afin d'avoir une idée globale sur l'effet de la température ambiante sur ces critères, les relations entre la TC et la TA, d'une part, et le $\mathrm{NH}$ et la TA d'autre part, ont été étudiées par analyse de variance. La variabilité individuelle a été prise en compte en introduisant l'animal en cofacteur.

La TC a été reliée à la TA par la relation :

$\mathrm{TC}=37,61( \pm 0,133)+0,139( \pm 0,004) * \mathrm{TA}$

$\left(\mathrm{R}^{2}=0,419, \mathrm{ETR}=0,37, \mathrm{n}=3061, \mathrm{p}<0,0001\right)$

Le $\mathrm{NH}$ a été relié à la TA par la relation :

Figure 5 : variation du niveau d'hyperventilation $(\mathrm{NH})$, de la température ambiante (TA) et de l'humidité relative (HR) au cours de la journée. Moyenne des données de tous les jours de mesures de NH (jours 32, 34, 36, 38, 40, 41).

$\mathrm{NH}=-244,1( \pm 10,8)+12,1( \pm 0,359) * \mathrm{TA}$

$\left(\mathrm{R}^{2}=0,435, \mathrm{ETR}=33,5, \mathrm{n}=2494, \mathrm{p}<0,0001\right)$

$\mathrm{Au}$ cours de cette expérience aucun coup de chaleur aigu n'a été observé, ni d'après les paramètres climatiques, ni d'après les données physiologiques recueillies. Seulement quatre animaux expérimentaux sont morts : en première semaine un mâle dans le cercle 2, en deuxième semaine un mâle et une femelle dans le cercle 2, et un mâle dans le cercle 3. Les causes de ces mortalités n'ont pas été élucidées mais elles sont intervenues après plusieurs jours de dépérissement, ce qui exclut l'hypothèse d'une mort par coup de chaleur. Il n'y a pas eu de mortalité massive ni de pathologie enregistrées dans le reste du bâtiment pendant l'expérimentation.

\section{Effet du sexe sur les paramètres évalués}

Le poids moyen des animaux à 42 jours a été de $2129 \pm 27 \mathrm{~g}$, avec une différence significative ( $p<0,001)$ entre mâles $(2311$ $\pm 28 \mathrm{~g}$ ) et femelles $(1953 \pm 33 \mathrm{~g})$. La consommation d'aliments a été significativement plus faible chez les femelles que chez les 
mâles (tableau I) sur l'ensemble des deux semaines d'expérimentation (de $391 \mathrm{~g}, \mathrm{p}=0,004)$. La différence n'a toutefois pas été significative pendant la $5^{\mathrm{e}}$ semaine $(\mathrm{p}=0,069)$, mais elle l'a été à la $6^{\mathrm{e}}$ semaine $(\mathrm{p}=0,002)$. De même, le gain de poids a logiquement été supérieur (de $218 \mathrm{~g}, \mathrm{p}=0,017$ ) chez les mâles sur la période complète, mais cette différence a été significative à la $5^{\mathrm{e}}$ semaine seulement $(\mathrm{p}=0,002)$, et non pas à la $6^{\mathrm{e}}$ semaine $(\mathrm{p}=0,098)$. L'indice de consommation n'a différé significativement ni sur chacune des deux semaines de l'essai, ni sur la période complète $(\mathrm{p}=0,644)$.

Sur l'ensemble des valeurs, il a été observé une tendance à une TC légèrement inférieure chez les femelles $\left(0,04{ }^{\circ} \mathrm{C}, \mathrm{p}=0,06\right)$, qui ont également eu un NH plus élevé (de 4,5 insp/min, $\mathrm{p}=0,009$ ).

\section{Interaction avec la localisation dans le bâtiment}

Le tableau II présente les différences de $\mathrm{TC}$, $\mathrm{NH}$ et de gain de poids (GP) en fonction du sexe et de la localisation des animaux dans le poulailler (cercles). Il présente également les valeurs de la TA et de l'HR pendant les mesures de la TC et du NH.

Les différences de GP entre les cercles n'ont pas été significatives à la $5^{\mathrm{e}}$ semaine $(\mathrm{p}=0,47)$. Elles ont en revanche été différentes à la $6^{\mathrm{e}}$ semaine $(\mathrm{p}=0,038)$, à cause notamment d'une faible valeur de la croissance des femelles dans le cercle 2, alors qu'il n'y a pas eu de différence entre cercles pour les mâles. Sur l'ensemble de l'expérimentation, les différences entre cercles n'ont pas été significatives $(p=0,08)$. Les mâles de ce même cercle 2 n'ont pas connu la même dépression de croissance que les femelles, avec un GP intermédiaire entre ceux des cercles 1 et 3 . La variation des performances de GP a été dans tous les cas supérieure dans le cercle 2 comparé aux autres cercles, y compris pour les mâles.

Les différences de TC entre les cercles ont été très significatives dans les deux périodes. Les TC des animaux du cercle 2 ont été significativement plus élevées que celles des autres cercles. Ce résultat peut être rapproché de la mesure de la TA du cercle 2, les jours de mesure de la TC, qui a également été supérieure à celle relevée dans les autres cercles. Les femelles du cercle 2 en particulier ont toujours eu une TC supérieure à celles des cercles 1 et 3 , ce qui peut être mis en relation avec la TA supérieure, mais aussi avec les performances de GP inférieures. Pour les mâles, la TC observée dans le cercle 1 a été significativement plus basse que dans les cercles 2 et 3 .

Le $\mathrm{NH}$ a été très significativement différent entre les cercles $(\mathrm{p}<$ $0,001)$. L'effet du sexe $(p=0,010)$ et l'interaction entre les deux $(\mathrm{p}=0,003)$ ont également été significatifs. Sur l'ensemble de la période expérimentale, les animaux du cercle 3 ont eu des $\mathrm{NH}$ supérieurs à ceux des autres cercles. A la $5^{\mathrm{e}}$ semaine le $\mathrm{NH}$ du cercle 1 a été significativement plus bas que ceux des cercles 2 et 3 , alors qu'à la $6^{\mathrm{e}}$ semaine les tendances ont été moins nettes.

Les mâles ont eu des NH inférieurs à ceux des femelles. En revanche, dans le cercle 2 en période deux, le NH des femelles a été significativement plus faible que celui des autres cercles. Pour les mâles, c'est dans le cercle 3 que les valeurs ont été les plus élevées.

\section{DISCUSSION}

\section{Intérêt du dispositif expérimental}

La méthodologie utilisée dans cette étude a permis la réalisation de mesures répétées de la TC et du NH directement dans des élevages commerciaux. Les animaux expérimentaux étaient dans des conditions très proches de ceux de l'élevage : l'origine des animaux, l'alimentation, la densité d'élevage et les conditions sanitaires étaient les mêmes. Ce type de dispositif permet donc de limiter au maximum les artefacts dus à des installations expérimentales généralement très différentes des conditions d'élevage réelles. Le stress infligé aux animaux était minimal et se réduisait aux mesures elles-mêmes, qui étaient très limitées dans le temps. Ce stress était bien moindre que celui subi lors de la nouvelle capture des animaux marqués libérés dans un bâtiment, comme décrit par de Basilio et coll. (8). Or les mesures de TC sont biaisées dès que l'animal est stressé par des manipulations trop brusques, comme par l'augmentation de l'activité physique (5). La mesure du NH n'est pas possible en mesures répétées avec des animaux en liberté au milieu d'un poulailler commercial (localisation des animaux, interaction avec le dérangement provoqué par la recherche des animaux). Ici, l'opérateur était situé à proximité du cercle pour les mesures du $\mathrm{NH}$ et dans le cercle pour les mesures de la TC, n'ayant qu'à prendre doucement l'animal au moment de mesurer la TC, ce qui ne biaise pas la mesure (10).

En outre, cette technique des cercles d'isolement permet d'étudier ou de contrôler l'effet de la localisation dans le bâtiment dont on voit dans la présente expérimentation qu'elle a eu un effet significatif sur la plupart des paramètres étudiés. Ainsi, dans les études sur l'effet de l'environnement de l'animal, il est possible de mesurer les paramètres d'ambiance à proximité directe des animaux expérimentaux. Ceci est d'autant plus utile dans des bâtiments de type ouvert, dans lesquels les conditions (TA, HR, vitesse de l'air) sont très différentes d'un endroit à l'autre.

Le nombre de cercles optimal dans un bâtiment dépend des paramètres étudiés : s'il s'agit exclusivement d'un suivi individuel des animaux, un nombre limité de cercles (trois par exemple) est suffisant. En revanche, pour des critères plus agrégatifs, comme la consommation alimentaire qui est mesurée par cercle (ou demi-cercle ici), l'unité expérimentale est le cercle et les trois répétitions de la présente expérience sont trop faibles pour mettre des effets en évidence : par exemple la différence d'indice de consommation (IC) entre mâles et femelles, entre 29 et 35 jours, a été ici non significative, alors que les valeurs absolues ont été de 1,78 (mâles) vs 1,93 (femelles), ce qui représentait une différence assez forte. La technique doit donc être adaptée à l'objet d'étude, mais la mise en œuvre est simple et peu onéreuse, et cela ne pose donc pas de problème particulier. En outre, l'adaptation de la technique par le cloisonnement en demi-cercles comme ici est une manière simple de multiplier les répétitions.

\section{Relations entre la TC, le NH et les paramètres climatiques}

Globalement les valeurs du $\mathrm{NH}$ et de la TC obtenues ont suivi les fluctuations de la TA et de l'HR (figures 4 et 5) d'une façon similaire aux observations obtenues en laboratoire par Zhou et Yamamoto (26). Toutefois, les courbes décrivant les paramètres climatiques et les paramètres animaux n'étaient pas strictement parallèles, signifiant que la réaction physiologique des animaux ne pouvait pas être décrite d'une façon satisfaisante par la simple donnée des mesures de la TA et de l'HR. Par exemple, entre 12 h 00 et 13 h 00, la TC a augmenté encore alors que la TA a stagné et que l'HR n'avait pas commencé à remonter. A l'inverse, la TC est descendue moins vite que la TA dans la seconde partie de la journée, aboutissant à $19 \mathrm{~h} 00$ à une valeur proche de celle de $9 \mathrm{~h} 00$, alors que la TA était presque de $3{ }^{\circ} \mathrm{C}$ audessous de sa valeur de $9 \mathrm{~h} 00$. Il en est de même pour le $\mathrm{NH}$ qui a augmenté également de 12 h 00 à 13 h 00, alors que la TA a diminué, et qui a retrouvé en fin de journée des valeurs proches de celles enre-

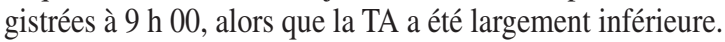

Bien que les observations précédentes montrent que les variations du NH, de la TC et de la TA ne sont pas strictement couplées, la relation générale existant entre ces paramètres a pu être étudiée. Dans plusieurs expérimentations publiées, il est possible de calculer 
l'incrément de la TC provoqué par l'augmentation de la TA. Par exemple, Deeb et Cahaner (12) trouvent une augmentation de 1,14 ${ }^{\circ} \mathrm{C}$ de la $\mathrm{TC}$ en passant de 22 à $32{ }^{\circ} \mathrm{C}$, soit $0,114{ }^{\circ} \mathrm{C}$ de la $\mathrm{TC}$ par degré de la TA. On retrouve des valeurs comparables chez de Basilio et coll. (11) où l'incrément varie de 0,087 à 0,142 selon les traitements (conditionnement thermique et alimentation), ou chez Yahav et Hurwit (24) avec une valeur de 0,11 pendant le conditionnement thermique chez des animaux plus jeunes (5 jours d'âge). Ici, le traitement statistique était différent puisqu'il ne s'agissait pas d'une expérience avec deux traitements à température fixe, mais d'une analyse statistique d'un grand nombre de mesures répétées. Ceci a permis de dégager une loi générale basée sur des données sur 120 animaux, 14 jours et plusieurs répétitions par jour. La valeur trouvée, $0,139{ }^{\circ} \mathrm{C}$ de TC par $1{ }^{\circ} \mathrm{C}$ de TA, a été sensiblement plus élevée que celles de la littérature, ce qui pouvait être dû aux conditions tropicales naturelles de l'essai, combinant une HR élevée à la TA.

Le même type d'approche a permis de calculer l'incrément du NH provoqué par l'augmentation d'un degré de la TA. Bien que les conditions ne soient pas exactement comparables, la valeur de 12,1 insp $/ \mathrm{min} /{ }^{\circ} \mathrm{C}$ obtenue ici peut être rapprochée des valeurs de l'ordre de 8 à $12 \mathrm{insp} / \mathrm{min} /{ }^{\circ} \mathrm{C}$ obtenues par Zhou et Yamamoto (26), ou El Hady et Sykes (13). Encore une fois, l'intérêt des présents résultats est leur mesure sur un grand nombre d'observations, dans des conditions réelles et sur une plage continue de températures.

Ces valeurs, obtenues dans des conditions où les animaux n'étaient pas en détresse physiologique, peuvent être considérées comme une réaction physiologique «normale » à la chaleur et être utilisées comme une base de comparaison permettant d'identifier des situations dans lesquelles les animaux souffrent davantage de la chaleur.

\section{Effets du sexe et de la localisation dans le bâtiment}

Le poids vif des animaux en fin de période expérimentale (2 $129 \mathrm{~g}$ ) correspondait à une valeur convenable pour un élevage industriel. On peut donc considérer que l'expérience s'est déroulée dans des conditions normales d'élevage. Les différences de gain de poids entre sexes ont été sensiblement moins élevées que dans les expériences rapportées par d'autres auteurs (19). En outre, elles ont été plus élevées dans la $5^{\mathrm{e}}$ semaine (123 g) que dans la $6^{\mathrm{e}}(97 \mathrm{~g})$, contrairement aux résultats d'Ozkan et coll. (21) qui obtiennent respectivement des différences de 40 et $70 \mathrm{~g}$ pour des poulets Cobb dans des conditions de laboratoire.

Pendant la période expérimentale, il n'y a pas eu de coup de chaleur provoqué par des TA supérieures à $36^{\circ} \mathrm{C}(7)$ et aboutissant à des mortalités importantes. Toutefois, on a observé des augmentations de $\mathrm{TC}$ supérieures à $0,3{ }^{\circ} \mathrm{C}$ et des $\mathrm{NH}$ supérieurs à $60 \mathrm{insp} / \mathrm{min}$, qui représentent les limites de la zone de thermoneutralité, définie par Arieli et coll. (2) chez la pondeuse. Contrairement aux observations de Teeter et coll. (22) qui situent le début du stress thermique à une TA de $32{ }^{\circ} \mathrm{C}$ chez le poulet de chair, cette étude a montré des effets sur la $\mathrm{TC}$ et le $\mathrm{NH}$ correspondant à des états de stress à partir d'une TA de $30^{\circ} \mathrm{C}$, avec une augmentation continue du $\mathrm{NH}$ au fur et à mesure de l'élévation de la TA. Les présents résultats en élevage commercial sont, en revanche, cohérents avec ceux de Zhou et coll. (25) résultant d'observations faites en laboratoire. Les présentes données ont permis de situer les moments de stress thermique au cours de la journée dans les conditions d'élevage, et en particulier de montrer la dissymétrie de la courbe d'évolution de la TC qui a montré une persistance des valeurs élevées dans l'après-midi, alors que la TA était déjà redescendue.

Les différences significatives des mesures effectuées dans les cercles positionnés à différents endroits du poulailler (centre, extrémités) ont montré que les conditions sensiblement différentes qui y régnaient ont eu un effet sur les performances. Les mesures de la TA ont montré une différence entre les cercles (TA supérieure dans le cercle 2 au centre du bâtiment), tandis que l'HR n'a pas été significativement différente entre les cercles. Les mesures d'HR ont été différentes entre les sexes, tant dans la moyenne générale des mesures (tableau II) que dans certaines périodes de la journée avec en particulier des valeurs supérieures près des mâles par rap-

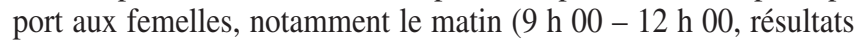
non présentés). Au-delà des artefacts expérimentaux, il est possible que ces différences aient été dues à des volumes d'excréta supérieurs chez les mâles, à une humidité supérieure des excréta (consommation d'eau) ou à un gaspillage d'eau supérieur. La mesure de la consommation d'eau serait une donnée importante à mesurer pour mieux comprendre les causes de ces observations. On peut en tout cas faire l'hypothèse que les animaux influencent les conditions climatiques à leur proximité immédiate.

De manière générale, il ne semble pas qu'il y ait eu dans cette étude une relation claire entre le GP et la TC, contrairement aux observations d'Ozkan et coll. (21). Il est toutefois remarquable que les femelles du cercle 2 , qui ont montré à la $6^{\mathrm{e}}$ semaine une forte dépression de croissance, ont eu au même moment une TC très supérieure à celles des cercles 1 et 3 . Dans les expériences d'acclimatation, il est classique de constater une température inférieure chez les animaux acclimatés, donc plus résistants (10). Ici les femelles du cercle 2 ont eu en même temps des valeurs de $\mathrm{NH}$ inférieures. On peut donc faire l'hypothèse que l'augmentation de la TC était davantage liée à un état pathologique qu'au seul stress thermique.

Johnson et MacLaury (16) avaient déjà montré une valeur du NH plus élevée chez les femelles que chez les mâles (+ 4 insp/min), comparable aux présents résultats $(+4,25 \mathrm{insp} / \mathrm{min})$. Les différences du $\mathrm{NH}$ entre mâles et femelles ont été plus importantes en début de stress thermique que lors du pic (données non présentées). Il semble que les femelles aient réagi plus vite à l'élévation de la TA en augmentant leur NH dès les premières heures de la matinée. A partir du moment du pic thermique, les NH des mâles et des femelles se sont rejoints. D'autres expériences, comme celles de May et Lott (20), suggèrent que ces différences entre sexes sont liées à la capacité d'adaptation à la chaleur. Ces auteurs observent une meilleure adaptation des femelles, dont l'IC ne se détériore que de 0,13 unité quand la TA passe de 22 à $30^{\circ} \mathrm{C}$, alors que celui des mâles augmente de 0,33 unité dans les mêmes conditions. Les différences entre sexes constatées sur le NH ne semblent pas aussi claires sur la TC, tant dans la présente expérimentation que dans celle de Berrong et Washburn (3). La mesure du NH menée parallèlement à celle de la TC a permis une meilleure évaluation de l'état de stress thermique des poulets mâles et femelles.

La résistance à la chaleur a été souvent reliée à la différence de poids vif et de vitesse de croissance, comme cela a été par exemple relevé par Berrong et Washburn (3) qui ont observé des mortalités de 2 et 25 p. 100 respectivement chez des animaux à croissance rapide et lente (Athens-Canadian Randombred), lors d'un coup de chaleur à $38^{\circ} \mathrm{C}$.

Au-delà de la différence de poids vif, une explication possible de la mortalité supérieure des mâles (constatée dans les essais de terrain) pourrait être une différence de résistance à la chaleur due à des différences fonctionnelles entre sexes marquées ici par les valeurs supérieures du NH. Toutefois les données disponibles dans la littérature permettent difficilement de séparer les effets de ces deux facteurs. Des expériences permettant cette distinction permettraient de mieux comprendre le lien entre la réaction à la chaleur, l'intensité de la croissance et les différences physiologiques directement liées au sexe. 


\section{CONCLUSION}

Il existe des variations de la TA entre l'extérieur et l'intérieur des bâtiments, et à l'intérieur d'un même bâtiment. Il est nécessaire de mesurer les paramètres climatiques en plusieurs points au niveau des animaux pour pouvoir interpréter l'effet de la chaleur sur la production. Il serait également important de mesurer les paramètres liés à la ventilation (17) qui peuvent générer une hétérogénéité des conditions dans le bâtiment.

Il est possible de mettre en évidence le niveau de stress thermique du poulet de chair en élevage commercial à travers les mesures de la $\mathrm{TC}$ et du NH. Ces paramètres dépendent des conditions climatiques, et notamment de la TA et de l'HR, mais ne varient pas de façon strictement parallèle au climat. Les variations du NH étant plus brutales que celles de la TC, les informations apportées par les deux mesures sont complémentaires.
L'observation, dans les élevages, de la plus grande sensibilité des mâles aux coups de chaleur peut en partie s'expliquer par leur TC plus élevée, leur NH plus faible et leur moindre capacité de réaction aux conditions climatiques. Les différences entre sexes pourraient augmenter avec le niveau de stress.

Enfin, la méthodologie d'expérimentation in situ mise en oeuvre dans cette expérimentation a permis d'obtenir des résultats expérimentaux dans des conditions très proches de l'élevage réel. Ceci est extrêmement utile pour éviter des biais expérimentaux, notamment dans l'estimation des conditions climatiques, fortement liées à l'environnement immédiat de l'animal, qui ne peut pas être reproduit précisément au laboratoire.

\section{Remerciements}

Ce travail a été réalisé grâce au soutien du programme Ecos/Anuies et du projet Fonacit-Ucv-Inia n ${ }^{\circ} 2005000420$.

\section{BIBLIOGRAPHIE}

1. ANGULO I., 1991. Manejo nutricional de aves bajo condiciones de estrés térmico. Fonaiap Divulga (Julio-Septiembre): 2-4.

2. ARIELI A., MELTZER A., BERMAN P., 1980. The thermoneutral zone and seasonal acclimatisation in the hen. Br. Poult. Sci., 21: 471-478.

3. BERRONG S., WASHBURN K., 1998. Effects of genetic variation on total plasma protein, body weight gains, and body temperature responses to heat stress. Poult. Sci., 77: 379-385.

4. BOUVAREL I., FRANCK Y., DE SAINT JAN B., GUILLAUMIN J.M., GERAERT P.A., RUDEAUX F., FERCHAL E., ROFFIDAL L., ECKENFELDER B., 1997. Utilisation de la mise à jeun pendant la période estivale. Journ. Rech. Avic., 2 : 165-168.

5. DAWSON W.R., WHITTOW G.C., 2000. Avian physiology: Regulation of body temperature, 5th Edn. New York, NY, USA, Academic Press.

6. DE BASILIO V., 1999. L'acclimatation précoce et I'alimentation alternée augmentent la résistance des poulets de chair mâles soumis à un stress thermique. Mémoire DEA, Ecole nationale supérieure agronomique, Rennes, France, $23 \mathrm{p}$.

7. DE BASILIO V., 2002. Acclimatation précoce des poulets de chair au climat tropical. Thèse Doct. Sci., Ecole nationale supérieure agronomique, Rennes, France, $147 \mathrm{p}$.

8. DE BASILIO V., OLIVEROS I., VILARINO M., DIAZ J., LEON A., PICARD M., 2001. Intérêt de l'acclimatation précoce dans les conditions de production des poulets de chair au Venezuela. Revue Elev. Méd. vét. Pays trop., 54 : 159-167.

9. DE BASILIO V., PICARD M., 2002. La capacité de survie des poulets à un coup de chaleur est augmentée par une exposition précoce à une température élevée. Prod. Anim., 15 : 235-246.

10. DE BASILIO V., REQUENA F., LEON A., VILARINO M., PICARD M., 2003. Early-age thermal conditioning immediately reduces body temperature of broiler chicks under a tropical environment. Poult. Sci., 82: $1235-1242$

11. DE BASILIO V., VILARINO M., YAHAV S., PICARD M., 2001. Early age thermal conditioning and a dual feeding program for male broilers challenged by heat stress. Poult. Sci., 80: 29-36.

12. DEEB N., CAHANER A., 2001. Genotype by environment interaction with broiler genotypes differing in growth rate. 2. The effects of high ambient temperature on Dwarf versus normal broilers. Poult. Sci., 80: 541-548.
13. EL HADY H., SYKES A., 1982. Thermal panting and respiratory alkalosis in the laying hen. Br. Poult. Sci., 23: 49-57.

14. FAO, 2006. Statistical databases. http://faostat.fao.org

15. FENAVI, 2005. Material divulgativo. In: IX Congreso Nacional de Avicultura, 11-14 mayo 2005. Caracas, Venezuela, FENAVI, 5 p.

16. JOHNSON T., MACLAURY D., 1974. Respiratory frequency in birds selected for high and low oxygen consumption. Poult. Sci., 53: 16191620.

17. LOTT B., SIMMONS J., MAY J., 1998. Air velocity and high temperature effects on broiler performance. Poult. Sci., 77: 391-393.

18. MC DONALD K., BELAY T., DEYHIM F., TEETER R., 1990 Comparison of the 5-day acclimation period and fasting techniques to reduce broiler heat distress mortality. Poult. Sci., 69: 90.

19. MC LEAN J., SAVORY C., SPARKS N., 2002. Welfare of male and female broiler chickens in relation to stocking density, as indicated by performance, health and behaviour. Anim. Welfare, 11: 55-73.

20. MAY J., LOTT B., 2001. Relating weight gain and feed: gain of male and female broilers to rearing temperature. Poult. Sci., 80: 581-584

21. OZKAN S., AKBAS Y., ALTAN O., ALTAN A., AYHAN V., OZKAN K., 2003. The effect of short-term fasting on performance traits and rectal temperature of broilers during the summer season. Br. Poult. Sci., 44: 88-95.

22. TEETER R., 1994. Optimizing production of heat stressed broilers. Poult. Dig., 94: 10-27.

23. TEETER R., WIERNUSZ C., BELAY T., SMITH M., 1989. Broilers exposed to acute heat distress are manageable. Feedstuffs (Oct.): 18-25.

24. YAHAV S., HURWIT Z., 1996. Induction of thermotolerance in male broiler chickens by temperature conditioning at early age. Poult. Sci., 75 402-406.

25. ZHOU W., CHAIYABURT N., FUIITA M., YAMAMOTO S. 1999. Distribution of body fluid and change of blood viscosity in broilers (Gallus domesticus) under high temperature exposure. J. therm. Biol., 24 193-197.

26. ZHOU W., YAMAMOTO S., 1997. Effects of environmental temperature and heat production due to food intake on abdominal temperature, shank skin temperature and respiration rate of broilers. $B r$ Poult. Sci., 38: 107-114.

Reçu le 06.07.2005, accepté le 04.12.2006 


\section{Summary}

Pérez M., De Basilio V., Colina Y., Oliveros Y., Yahav S., Picard M., Bastianelli D. Assessment of the Level of Heat Stress in Broilers by Measuring Body Temperature and Hyperventilation under Poultry Farm Conditions in Venezuela

Repeated measurements on poultry are difficult to perform in real production conditions because of the difficulty to identify and retrieve the animals. A monitoring technique by which small groups of animals are isolated in wire fence circles within the poultry shed is proposed. This method was used in a production poultry farm in Venezuela to determine variations in body temperature (BT) and the level of hyperventilation $(\mathrm{LH})$, based on the location in the poultry shed and the sex of the animals. Three wire fence circles were set up and divided into two parts, each half-circle containing 20 male or female broilers. From day 29 of age, the ambient temperature (AT) and relative humidity (RH) were continuously registered, and BT (in terminal colon) and LH were recorded every hour from 9:00 to 19:00 every other day for two weeks. The measurements of BT and $\mathrm{LH}$ varied during the day, without being completely parallel to the climatic parameters AT and $\mathrm{RH}$. There were significant differences between circles for all parameters measured. The effect of sex was not significant on BT ( $p=0.06)$ but was significant on LH $(p=0.01)$ and the interaction with the circle location $(p<0.001$ for BT and $p$ $<0.01$ for LH). Sex had a significant effect on feed consumption $(p<0.01)$ and weight gain $(p=0.02)$ but not on the food conversion ratio $(p=0.64)$. Climatic measurements were also different between circles and between sexes, with in particular a higher RH near males $(p<0.001)$. Of the overall dataset, BT and $\mathrm{LH}$ increased by $0.139^{\circ} \mathrm{C}$ and $12.1 \mathrm{insp} / \mathrm{min}$, respectively, for each $1{ }^{\circ} \mathrm{C}$ AT increase. It is concluded that climatic conditions show heterogeneity within the poultry shed, with consequences on the comfort of broilers, and that climatic parameters have to be completed by measurements on animals (BT and $\mathbf{L H}$ ) in order to characterize the heat stress. The experimental technique proposed here allows observing animals repeatedly in the real conditions of a poultry production farm.

Keywords: Broiler chicken - Tropical climate - Body temperature - Respiration rate - Poultry farming Measurement - Venezuela.

\section{Resumen}

Pérez M., De Basilio V., Colina Y., Oliveros Y., Yahav S., Picard M., Bastianelli D. Evaluación del nivel de estrés térmico mediante la medida de la temperatura corporal y del nivel de hiperventilación en el pollo de carne bajo las condiciones de producción en Venezuela

Las medidas repetidas efectuadas sobre aves de cría son difíciles de poner en marcha debido a la dificultad en la identificación y la recuperación de los animales. Se propone una técnica de seguimiento mediante el aislamiento de pequeños grupos de animales en círculos situados en el seno mismo de la cría. Este método fue utilizado en un gallinero de producción en Venezuela para determinar la variación de la temperatura corporal (TC) y del nivel de hiperventilación $(\mathrm{NH})$, en función de la localización en el edificio y del sexo de los animales. Se crearon tres círculos de cedazo de gallinero y se dividieron en dos, con 20 pollos hembras o machos en cada mitad de los círculos. A partir del día 29 de edad, se registraron la temperatura ambiente (TA) y la humedad relativa (HR) en forma continua y la TC (colon terminal) y el NH se midieron cada hora, de las 9:00 a las 19:00 hrs, día por medio y durante dos semanas. Las medidas de la TC y del $\mathrm{NH}$ variaron durante el día, sin ser rigurosamente paralelas a los parámetros climáticos TA y HR. Se observaron diferencias significativas entre los círculos para todos los parámetros medidos. El efecto del sexo no fue significativo para la TC $(p=0,06)$, pero si para el $\mathrm{NH}(p=0,01)$ y la interacción con la localización del círculo ( $p<0,001$ para la TC y $p<0,01$ para el $\mathrm{NH}$ ). El sexo presentó un efecto significativo sobre el consumo alimenticio $(p<0,01)$ y la ganancia de peso $(p=0,02)$, pero no para el índice de consumo $(p=0,64)$. Las medidas climáticas fueron igualmente diferentes entre los círculos y entre los sexos, principalmente con una HR más fuerte en los machos $(p<$ 0,001). Sobre el conjunto de datos, la TC y el $\mathrm{NH}$ aumentaron respectivamente de $0,139^{\circ} \mathrm{C}$ y de $12,1 \mathrm{insp} / \mathrm{min}$ por grado de aumento de la TA. Se concluye que las condiciones climáticas presentan una heterogeneidad en el seno del edificio, con consecuencias sobre el confort de los pollos, y que las medidas sobre los animales (TC y $\mathrm{NH}$ ) deben completar los datos climáticos para describir el estado del estrés térmico. La técnica experimental propuesta para permite la observación de animales de manera repetida bajo condiciones reales en un gallinero de producción.

Palabras clave: Pollo de engorde - Clima tropical Temperatura del cuerpo - Tasa de respiración - Cría de aves de corral - Medición - Venezuela. 